\title{
COLLISIONAL EXCITATION OF AUTOIONIZING LEVELS
}

\author{
by \\ Leo Goldbera * $\dagger$, Andrea K. Dupree * and John W. Allen (*) \\ (Harvard College Observatory * and Smithsonian Astrophysical Observatory $†$ Cambridge, Massachusetts, U. S. A.)
}

REsumé. - On montre que l'excitation par chocs des niveaux d'autoexcitation est un facteur important dans la déter. mination du taux d'ionisation de certains ions aux températures coronales. On a mené des calculs préliminaires pour $O I V, O V, O V I, F e X V$ et $F e X V I$. L'autoionisation semble importante pour $O I V$ et augmente d'un fac. teur 2 le taux d'ionisation de Fe XV et Fe XVI.

ABstraCt. - Collisional excitation of autoionizing levels is found to contribute significantly to the ionization rate for certain ions at temperatures characteristic of the solar corona. Preliminary calculations have been carried out fo $O I V, O V, O V I, F e X V$ and Fe XVI. Autoionization may be of importance for $O I V$ and increases by appro ximately a factor of two the ionization rate of Fe $X V$ and $X V I$.

Резюме. - Показано, что возбуждение ударами уровней самовозбуждения является важным фактором в определении степени ионизации некоторых ионов при корональных температурах. Ђыли проведены предварительные вычисления для O IV, O V, O VI, Fe XV и Fe XVI. Самоионизация по-видимому значительна для O IV и умножает на 2 степень ионизации Fe XV и Fe XVI.

The total rate coefficient for collisional ionization by removal of an electron with principal quantum number $n$, and contributions from different $l$-values, usually includes only collisions directly to the continuum. But electrons may also be lost by autoionization following the excitation of an innershell electron to a bound state possessing energy greater than that required to remove a valence electron. This process has rarely been taken into account in calculations of ionization equilibrium, although its contribution to the photoionization rate may sometimes equal or exceed that associated with bound-free transitions (DITCHBURN and ÖPIK, 1962).

We have made a preliminary study of the importance of the autoionization process in the chromosphere and corona by carrying out approximate calculations for a number of ions of oxygen and iron. For this purpose we adopted an expression given by Seaton (1964) for the rate of collisional excitation :

$$
\text { (1) } q_{i j}=1.70 \times 10^{-3} \frac{f_{i j}}{\frac{10}{\mathrm{~W}}} \frac{-\frac{5040 \mathrm{~W}}{\mathrm{~T}}}{\mathrm{~T}^{\frac{1}{2}}} \mathrm{P}\left(\frac{\mathrm{W}}{k \mathrm{~T}}\right)
$$

$$
\left(\mathrm{cm}^{3} \mathrm{sI}^{-1}\right) \text {, }
$$

where $f$ is the oscillator strength and $\mathrm{W}$ the excitation energy in electron volts of the $(i \rightarrow j)$ transition, $\mathrm{T}$ is the temperature, and $\mathrm{P}\left(\frac{\mathrm{W}}{k \mathrm{~T}}\right)$ is a function that has been tabulated by VAN REGEMORTER (1962). Excitation and ionization from the ground level only were included in the computations. The rate coefficient for autoionization is obtained by summation of $q_{i j}$ over all upper levels $j$ that lie above the first ionization limit and for which the selection rules governing autoionization are obeyed (CONDON and SHORTLEY, 1963). Therefore, if the ground level of the ion is denoted by 1 , the autoionizing rate coefficient, $q_{1}$, is given by :

$$
q_{1}=\sum_{j} q_{i j}
$$

The rate $q_{1}$ is to be compared with the corresponding rate $q\left(\mathrm{X}^{+m}\right)$ for the collisional ionization of an $m$-times ionized atom. According to BuRGess and Seaton (1964), the ionization rate may be approximated by

$$
\text { (2) } \begin{aligned}
q\left(\mathrm{X}^{+m}\right)= & 2.0 \times 10^{-8} \mathrm{~T}^{\frac{1}{2}} \\
& \times \sum_{n . l} \frac{\zeta_{m}(\dot{n l}) 10^{\frac{5010 \mathrm{I}_{m}(n l)}{\mathrm{T}}}}{\mathrm{I}_{m}^{2}(n l)}\left(\mathrm{cm}^{3} \mathrm{~s}^{-1}\right)
\end{aligned}
$$

where $\zeta_{m}(n l)$ is the number of electrons having principal quantum number $n$ and angular quantum number $l$, and $I_{m}(n l)$ is the ionization potential of an $(n l)$ electron in electron volts.

In order to evaluate $q_{1}$ from equation (1), we must know the energies of levels $j$ and the oscillator strengths $f_{i}$. The energy levels were derived 
from Hartree-Fock calculations performed on the IBM-7094 of the Harvard University Computation Center, utilizing a program written by Dr. Charlotte FrozSE. Various approximate methods were employed in the calculation of the oscillator strengths. The details of the computation for each ion are described below.

\section{IroN}

The ground configurations of the ions Fe IXFe XVI have outer electrons with $n=3$ and $l=0,1$. For Fe IX-XIV, the relevant transitions are of the form $3 s^{2} 3 p^{x} \rightarrow 3 s 3 p^{x} n p$ with $n \geqslant 3$. Calculations of the energy levels showed that the early series members lie below the first ionization limit; although most of the oscillator strength in a series is contained in the first few members, there can be a significant contribution to the autcionizing rate from the upper levels (see results for $\mathrm{O}$ IV below). For a first reconnaissance however, rate coefficients were obtained only for Fe XV and Fe XVI, whose doubly excited levels lie above the first ionization limit. The most important transitions are, for $\mathrm{Fe} \mathrm{XV}, 2 p^{6} 3 s^{2}$ ${ }^{1} \mathrm{~S}-2 p^{5} 3 s^{2} n d{ }^{1} \mathrm{P}^{0}$, and for Fe XVI, $2 p^{6} 3 s^{2} \mathrm{~S}-2 p^{5}$ $3 s n d{ }^{2} \mathrm{P}^{0}$, with $n \geqslant 3$. In an exact calculation, the energy levels and oscillator strengths are evaluated for each value of $n$ and the rate coefficients are then summed over the entire series. For the purposes of this preliminary investigation, however, it is thought sufficient to treat each series as a single line having the frequency of the first member with $n=3$, and an oscillator strength $f=0.8 x$, where $x$ is the number of jumping electrons. The factor 0.8 represents the approximate fraction of the total oscillator strength that resides in the lowest members of a transition array (Allen, 1960). Thus, $f_{i j}$ was assumed to be 4.8 for both $\mathrm{Fe} X V$ and Fe XVI. The effect of interactions between configurations in the same complex was not included in the Hartree-Fock calculations, although it occurs in both $\mathrm{Fe} \mathrm{XV}$ and XVI. The results of Fromst (1964) indicate that neglect of complex interaction will lead to an error of about $10 \%$ in the calculated energy ; at most, the error will be less than $20 \%$. The results of the energy calculations are given in Table $I$.

The ratio $q_{1} / q\left(\mathrm{X}^{+m}\right)$ is plotted as a function of the temperature in Figures $1 a$ and $1 b$. The arrows denote the temperature interval in which the fraction of iron in the stage of ionization considered is within a factor of 10 of its maximum value, according to the recent results of BURGESS and Staton (1964), which include the effects of dielectronic recombination. The two ionization rates are comparable; hence, the autoionization process should be included whenever the degree of ionization of Fe XV and Fe XVI is desired to within a factor of two or better.

\section{TABLE I}

\section{Results of Hartree-Fock Calculations for} Fe XV and Fe XVI *.

\begin{tabular}{|c|c|c|c|c|}
\hline IoN & \multicolumn{2}{|l|}{ TERM } & $\mathrm{E}\left(\mathrm{cm}^{-1}\right)$ & $\mathrm{E}(\mathrm{eV})$ \\
\hline \multirow{2}{*}{$\mathrm{Fe} X \mathrm{~V}$} & $2 p^{6} 3 s^{2}$ & ${ }^{1} \mathrm{~S}$ & 0 & 0 \\
\hline & $2 p^{5} 3 s^{2} 3 d$ & ipo & $6.47 \times 10^{6}$ & 802 \\
\hline $\mathrm{I}(3 s)$ & $2 p^{6} 3 s$ & ${ }^{2} \mathrm{~S}$ & $3.63 \times 10^{6}$ & 450 \\
\hline $\mathrm{I}(2 p)$ & $2 p^{5} 3 s^{2}$ & 2Po & $7.54 \times 10^{6}$ & 1170 \\
\hline \multirow[t]{3}{*}{ Fe XVI } & $2 p^{6} 3 s$ & ${ }^{2} \mathrm{~S}$ & 0 & \\
\hline & $2 p^{5} 3 s^{2}$ & ${ }^{2} \mathbf{P} o$ & $5.81 \times 10^{6}$ & 72 \\
\hline & $2 p^{5} 3 s \quad 3 d$ & ${ }^{2} \mathrm{Po}^{\mathrm{o}}$ & $6.44 \times 10^{6}$ & 79 \\
\hline $\mathrm{I}(3 s)$ & $2 p^{6}$ & ${ }^{1} \mathrm{~S}$ & $3.91 \times 10^{6}$ & 48 \\
\hline $\mathbf{l}(2 p)$ & $2 p^{5} 3 s$ & 3.1 Po & $9.80 \times 10^{6}$ & 121 \\
\hline
\end{tabular}

(*) The energies of excitation and ionization are given relative to the ground states of the respective ions.

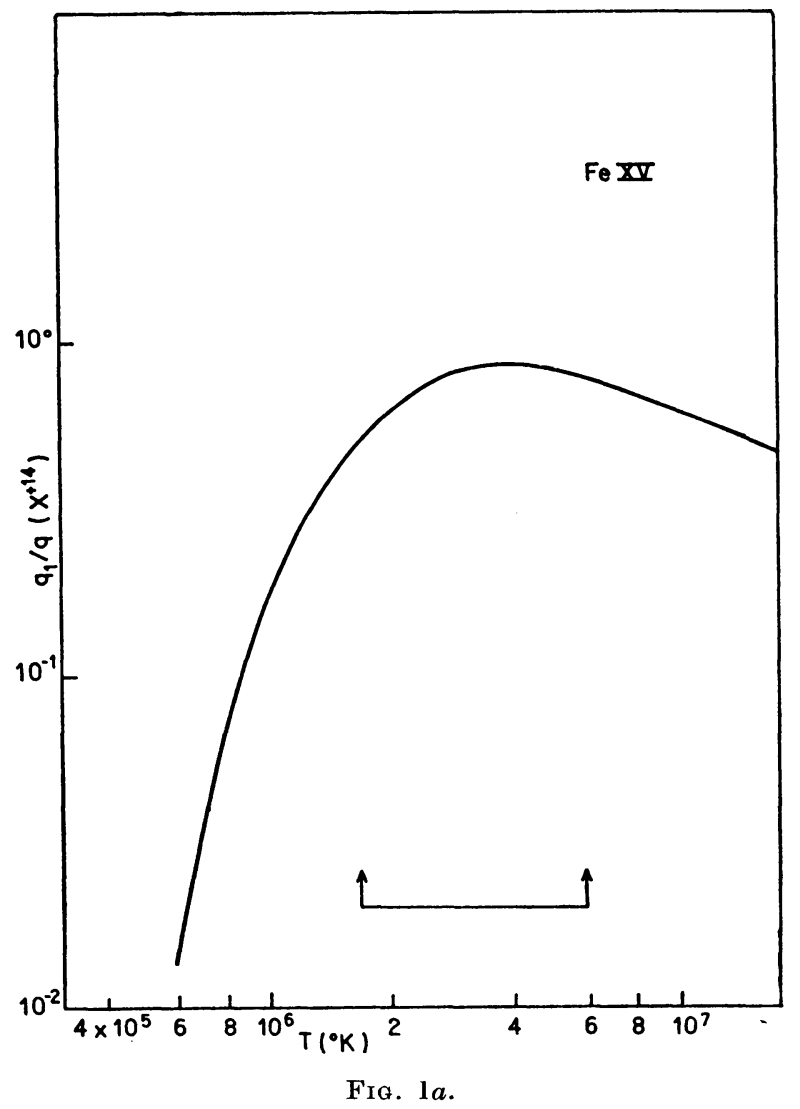




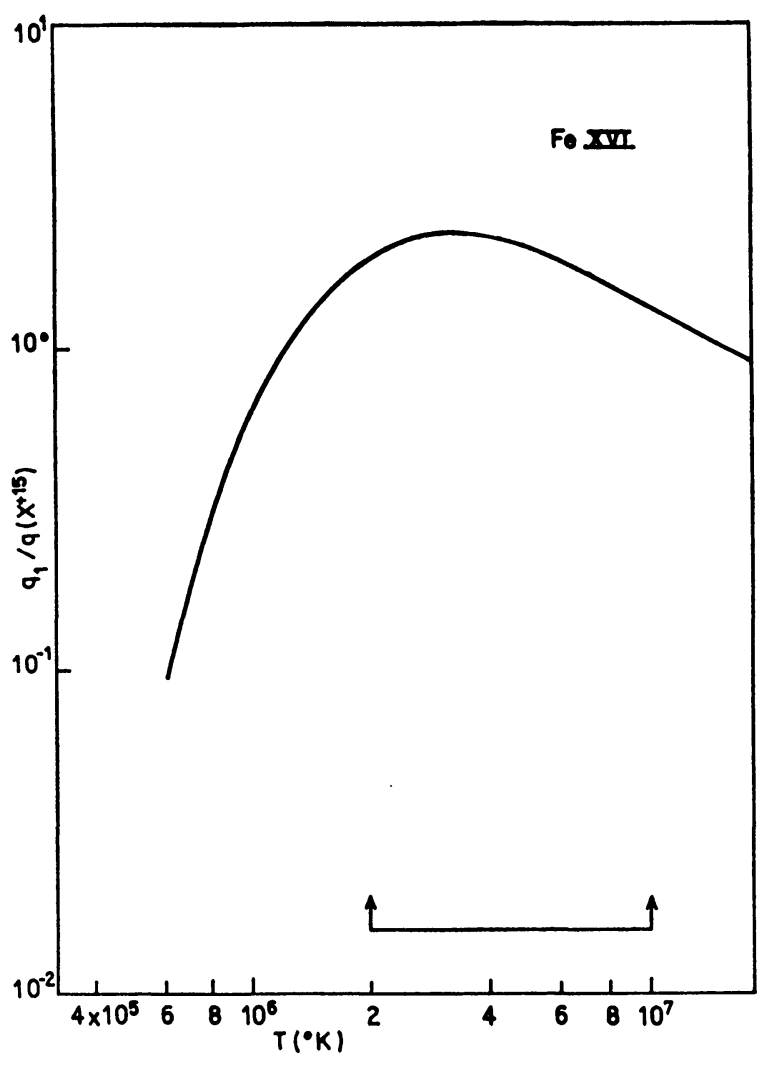

Fia. 1b.,

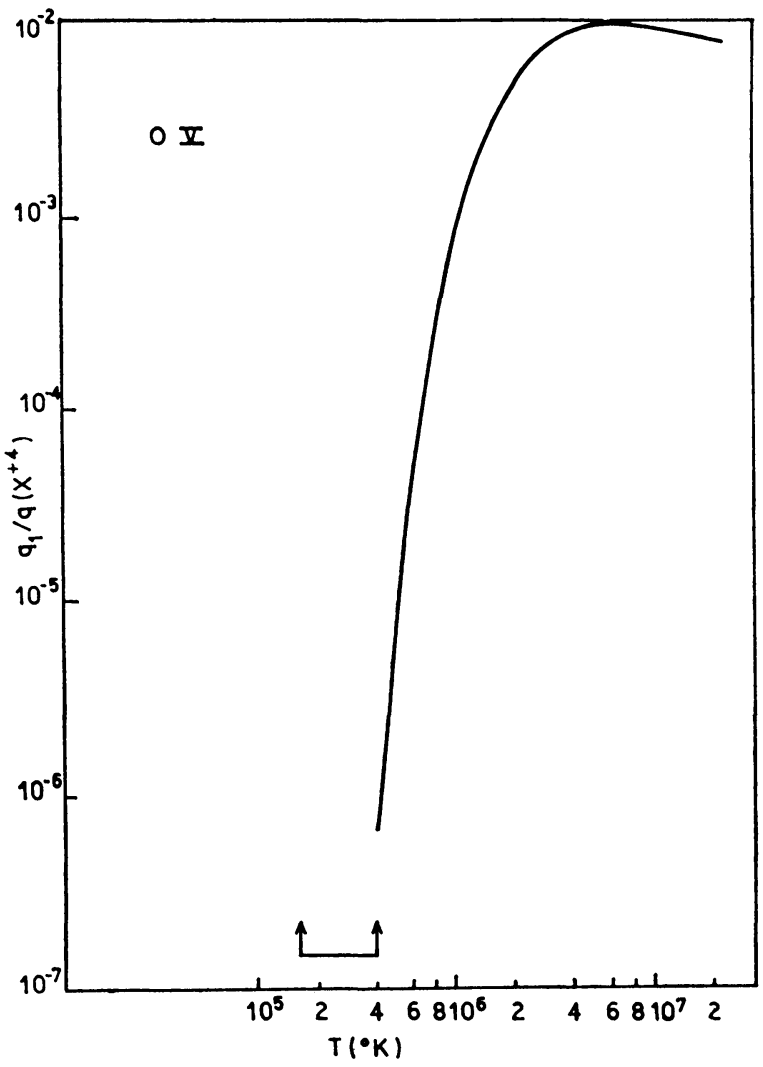

Fıa. $1 d$.

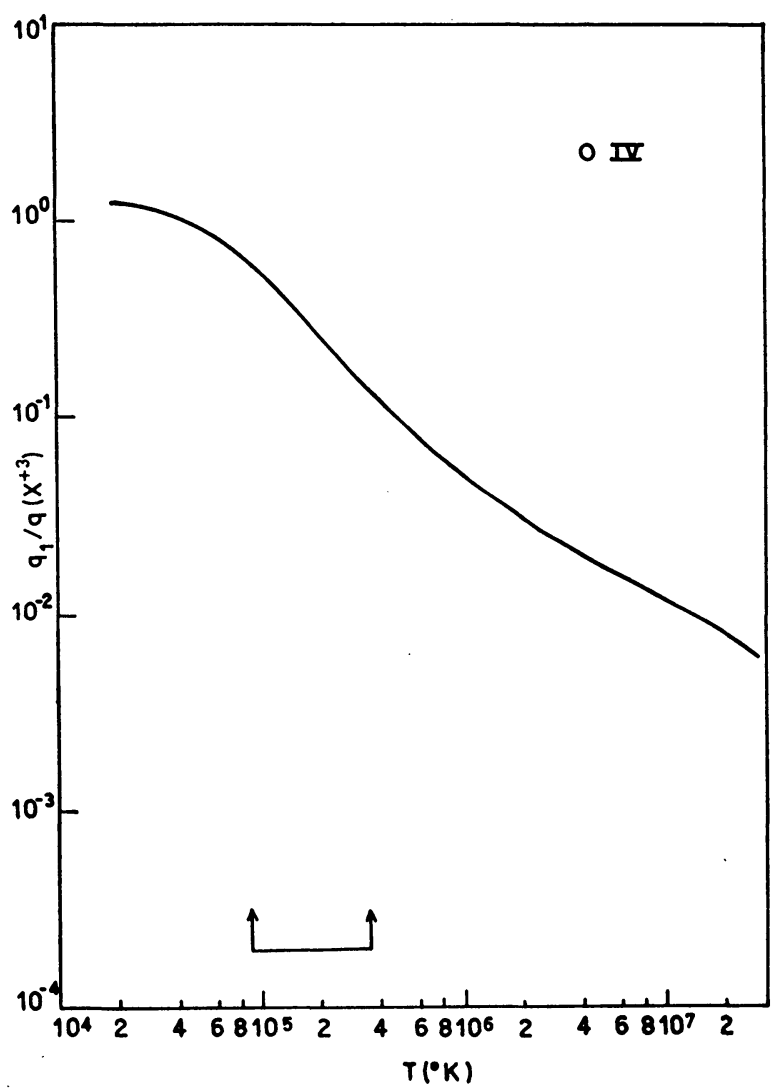

Fra. 1c.

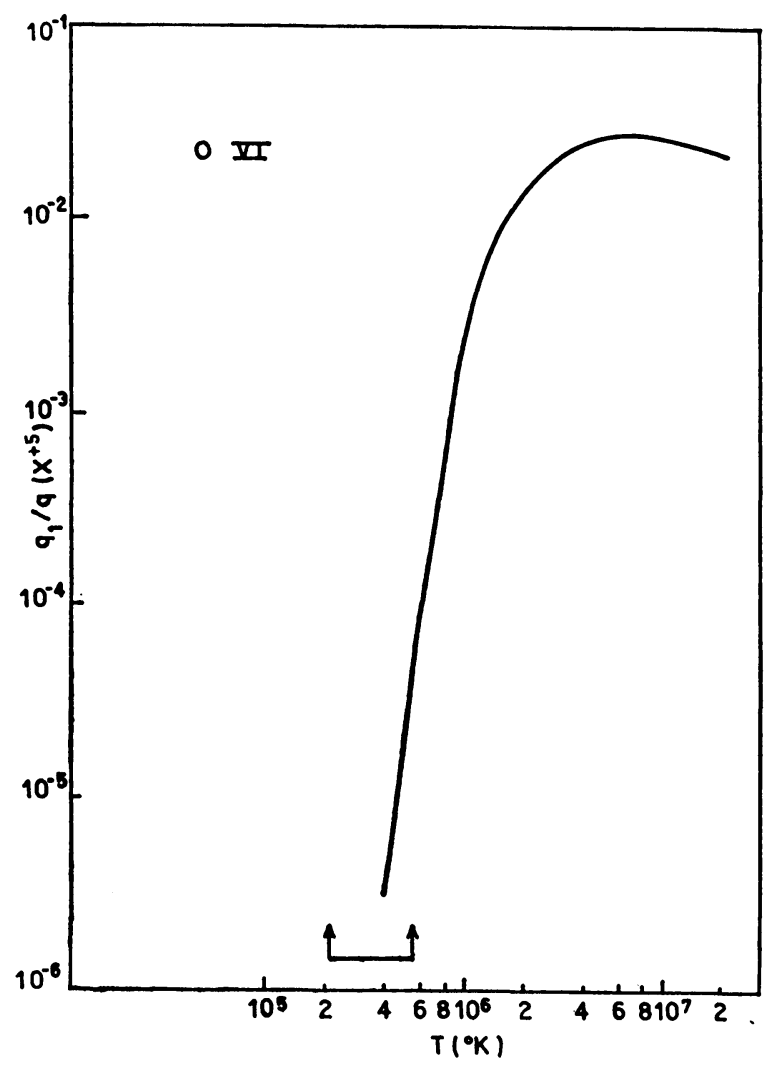

Fia. Ie.

The ratio of collisional excitation to collisional ionization as a function of $T$. Arrows indicate the temperature range over which the abundance of the ion is within a factor of 10 of its maximum value. The process of dielectronic recombination has been included in the calculations of ionization equilibrium. 


\section{OXYGEN}

The collisional excitation rates $q_{1}$ were calculated from equation ( 1 ) for the following transitions in $\mathrm{OIV}, \mathrm{OV}$ and $\mathrm{O} \mathrm{VI}$ :

$$
\begin{aligned}
\text { O IV } 1 s^{2} 2 s^{2} 2 p{ }^{2} \mathrm{P}_{0} \rightarrow & 1 s^{2} 2 s 2 p\left({ }^{3} \mathrm{P}\right) n p{ }^{2} \mathrm{D},{ }^{2} \mathrm{~S}, \\
& \\
& 18^{2} 282 p\left({ }^{1} \mathrm{P}\right) n p{ }^{2} \mathrm{D},{ }^{2} \mathrm{~S}, \\
n=4,5,6 &
\end{aligned}
$$

$0 \mathrm{~V} 18^{2} 2 s^{2}{ }^{1} \mathrm{~S} \rightarrow 1828^{2} n p^{1} \mathrm{P}^{0} \quad n=2,3,4,5,6$.

$0 \mathrm{VI} 1 s^{2} 2 s^{2} \mathrm{~S} \rightarrow 1 s 2 s\left({ }^{3} \mathrm{~S}\right) n p{ }^{2} \mathrm{Po} \quad n=2,3,4,5,6$.

The early members of the series $1 s^{2} 2 s 2 p n p$ in $O$ IV lie below the first ionization limit and therefore play no part in the autoionization process. The ${ }^{2} \mathrm{P}$ levels in $\mathrm{O}$ IV are not subject to autoionization because of the parity rule. The rate $q_{1}$ is to be compared with the corresponding rate $q\left(\mathrm{X}^{+m}\right)$, equation (2), for the collisional ionization of the $2 s$ and $2 p$ electrons in $\mathrm{OIV}$ and the $1 s$ and $2 s$ electrons in $\mathrm{OV}$ and $\mathrm{O} \mathrm{VI}$. The following details of the computations may be of interest.

For $O$ IV, radial wave functions of the terms $1 s^{2} 2 s 2 p\left({ }^{3} \mathrm{P}\right) n p 4 \mathrm{D}$ were used to approximate the wave functions of the ${ }^{2} S$ and ${ }^{2} D$ terms. The total energies obtained in this way were found to be 3-4 \% lower than the few experimental values that were available (MOORE, 1949). Hence, all calculated energies were increased by this small amount. For O VI, wave functions and energy levels derived for the series $1 s 2 s\left({ }^{3} \mathrm{~S}\right) n p{ }^{4} \mathrm{P}^{0}$ were used as approximations for the corresponding $n p{ }^{2} \mathrm{P}^{0}$ series. Values of $\sigma^{2}$ were calculated from the radial wave functions for transitions from the ground state to doubly-excited levels; f-values for these transitions were found from $\sigma^{2}$ with the aid of tables given by Shore and Menzel (1965).

TABLE II

Results of Hartree-Fook Calculations for O IV, V, VI.

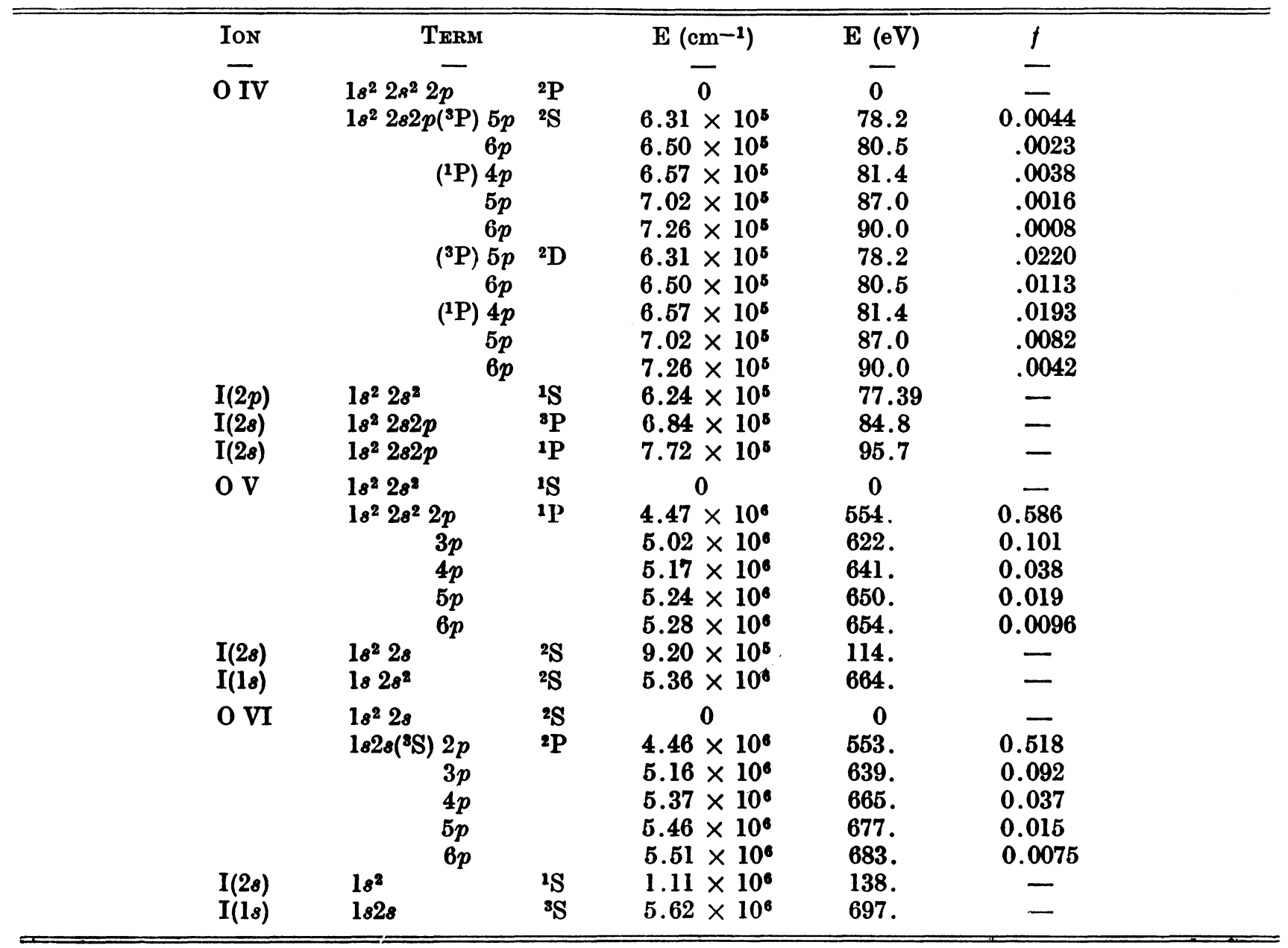


The results of the calculations of energy levels and oscillator strengths for oxygen are given in Table II. Finally, the ratios $q_{1} / q(\mathrm{X}+m)$ are shown as a function of temperature in Figures $1 c, 1 d, 1 e$. The arrows indicate the temperature range in which the oxygen ion has an abundance within a factor of ten of its maximum value. The calculations of ionization equilibrium for oxygen include dielectronic recombination, (ALLEN, 1964).

Note that the autoionization rate for $\mathrm{O} I V$ is comparable to the collisional ionization rate even though the three strongest members of the series are omitted because their energies are less than the ionization energy of 0 IV. In other ions also (for instance, Fe IX-XIV), excitation of an inner shell electron to the lowest levels of a transition array may require less energy than that needed for ionization of a valence electron. The results for O IV demonstrate the necessity of considering contributions from transitions which do not have the largest f-values in an array.

It does not appear that collisional autoionization processes will be important for $\mathrm{O} \mathrm{V}$ and $\mathrm{O} \mathrm{VI}$ at temperatures for which the ion is most abundant. The energies required to excite an electron from a $1 s$ shell are so large that the ratio $q_{1} / q(\mathrm{X}+m)$ attains a maximum only for extremely high temperatures and even then its value is considerably less than one.

Further investigation should be made of the location of doubly-excited levels in other ions; f-values for transitions to these levels are also extremely desirable. The presence of autoionization processes directly affects calculations concerned with ionization equilibrium in the solar chromosphere, corona, and other astrophysical applications.

Grateful acknowledgement is made of the advice of Dr. B. W. Shore on various aspects of the Hartree-Fock calculation. This work was supported in part by Grant NsG-438 from the National Aeronautics and Space Administration.

Manuscrit reçu le 7 mars 1965.

\section{BIBLIOGRAPHY}

Allen C. W., 1960, M. N., 121, 299.

ALLIEN J. W., 1964, private communication.

Burarss A. and Shaton M. J., 1964, M. N., 127, 335.

Condon E. M. and ShorTley G. H., 1963, The Theory of Atomic Spectra (Cambridge, England : Univer. sity Press).

DitohburN R. W. and ÖpIK U., 1962, in Atomic and Molecular Processes, ed. D. R. Bates (New York : Academic Press), chap. 3.
Fromse C., 1964, Ap. J., 140, 361.

Moore C. E., 1949, Atomic Energy Levels, N. B. S. Circular No. 467.

Seaton M. J., 1964, Plan. and Sp. Sci., 12, 55.

Shore B. W. and Menzer D. H., 1965, Ap. J. Suppl., 106.

VAN Reghmorter H., 1962, Ap. J., 136, 906.

\section{Discussion}

M. J. Seaton. - Whereas allowance for resonance processes increases recombination rates by one or two orders of magnitude, the effect on excitation or ionisation, will, I believe, be much less.

L. GoldBerG. - I agree that excitation to autoionizing levels will not, in most cases, increase ionization rates by as much as an order of magnitude. The importance of the iffect will vary from one ion to another, but the calculations presented here show that increases in ionization rates by a factor of two or three are quite possible.

A. BURaEss. - I think the inner-shell excitation cross-sections should perhaps be compared with innershell ionization cross-sections. The latter have been included in existing ionization balance calculations. For $\mathrm{Li}$, my estimate of $\mathrm{Q}_{e}=\sum_{n=2}^{\infty} \mathrm{Q}\left(1 s^{2} 2 s \rightarrow 1 s 2 s n p\right)$ is smaller than, but comparable with the innershell ionization cross-section.

L. GoLDBERG. - The calculations I have presented show that at least for some ions the inner-shell excitation cross-sections are substantially greater than the corresponding inner-shell ionization cross-sections, even though this may not be true for lithium. 